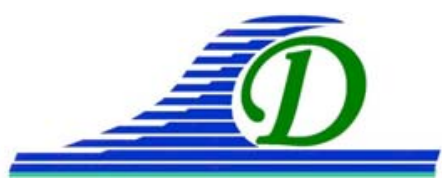
XIII ${ }^{\text {èmes }}$ Journées Nationales Génie Côtier - Génie Civil
Dunkerque, 2-4 juillet 2014

DOI:10.5150/jngcgc.2014.022 @ Editions Paralia CFL

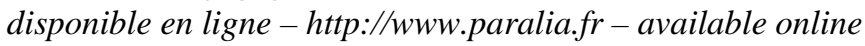

\title{
Analyse régionale des surcotes extrêmes de pleine mer et caractérisation de la dépendance spatiale
}

\author{
Jérôme WEISS ${ }^{1,2}$, Pietro BERNARDARA ${ }^{1,3}$, Michel BENOIT ${ }^{1,2}$
}

\author{
1. Laboratoire d'Hydraulique Saint-Venant (ENPC, EDF R\&D, CEREMA), \\ Université Paris-Est, 6 quai Watier, 78400 Chatou, France. \\ 2. EDF R\&D Laboratoire National d'Hydraulique et Environnement (LNHE), \\ 6 quai Watier, 78400 Chatou, France. \\ 3. EDF Energy R\&D UK Centre, 52 Grosvenor Gardens, London SW1W 0AU, UK.
}

\section{Résumé :}

Afin de prévenir le risque de submersion marine en zone côtière, la caractérisation probabiliste des phénomènes océano-météorologiques extrêmes est un élément fondamental. Par exemple, le concept de niveau de retour est fréquemment utilisé dans l'estimation des conditions hydrauliques ou océano-météorologiques (niveau marin, conditions de vagues) entrant dans le dimensionnement des ouvrages de protection côtière. Ces niveaux de retour sont habituellement estimés par des analyses statistiques locales, à partir des données uniquement observées au site d'intérêt. Cependant, le caractère limité des durées d'observation peut se traduire par une incertitude importante sur les niveaux de retour d'intérêt.

Par rapport à une analyse locale, l'analyse régionale permet de réduire ces incertitudes, en exploitant l'information d'un ensemble de sites dont les caractéristiques statistiques des extrêmes sont similaires. Dans une région dite homogène, la loi de probabilité des extrêmes est supposée identique en chaque site, à un indice local près. En particulier, les extrêmes observés en différents sites d'une région sont rassemblés dans un échantillon régional, celui-ci servant à estimer les paramètres de la loi régionale de distribution des extrêmes. Cependant, le fait de regrouper les observations requiert un traitement approprié de la dépendance spatiale. Cette étude propose de modéliser la dépendance spatiale dans le cadre de l'analyse régionale, en se basant sur la tendance qu'ont les sites à avoir un comportement similaire pendant une tempête. Le modèle obtenu permet par exemple d'estimer la durée effective de l'échantillon régional, ou encore de comparer la période de retour locale et régionale d'une tempête.

Un exemple d'application est donné à partir d'une base de données de surcotes de pleine mer observées en 67 sites équipés de marégraphes sur les côtes françaises, britanniques et espagnoles de l’Océan Atlantique, de la Manche et de la Mer du Nord.

Mots-clés : Génie côtier, Niveaux marins extrêmes, Surcotes de pleine mer, Valeurs extrêmes, Analyse régionale, Dépendance spatiale, Océan Atlantique, Manche, Mer du Nord 


\section{Thème 1 - Hydrodynamique côtière}

\section{Introduction}

Une protection efficace des zones côtières contre le risque de submersion marine nécessite de caractériser les événements océano-météorologiques extrêmes. En ingénierie côtière, le niveau de retour associé à une période de retour donnée est traditionnellement estimé par une analyse statistique locale, à partir de données provenant d'un site d'observation unique. Une limitation courante avec cette méthode est la durée d'observation, généralement trop courte pour estimer le niveau de retour avec précision.

L'analyse régionale est une solution possible pour réduire ces incertitudes (HOSKING \& WALLIS, 1997). Elle vise à tirer parti de l'information partagée par un ensemble de sites statistiquement similaires. L'hypothèse fondamentale est l'existence d'une loi régionale des extrêmes commune à tous les sites d'une région dite homogène, à un indice local près. En particulier, une manière d'estimer les paramètres de la loi régionale est la méthode du pooling, qui regroupe les extrêmes observés en différents sites d'une région dans un même échantillon régional (e.g., BERNARDARA et al., 2011).

Cependant, compte tenu de l'extension spatiale des tempêtes à l'origine des extrêmes observés, les effets de la dépendance spatiale doivent être étudiés lorsque cette méthode est utilisée. Par exemple, la durée effective de l'échantillon régional, obtenu en regroupant les observations de plusieurs sites, varie selon le degré de dépendance spatiale des sites de la région. Cette durée est requise pour estimer des périodes de retour, ou encore pour quantifier la plus-value d'une analyse régionale par rapport à une analyse locale.

Par ailleurs, le lien entre période de retour régionale et locale d'une tempête est une question peu étudiée dans la littérature scientifique. Par exemple, une tempête dont la période de retour est de 100 ans dans une région donnée ne va très probablement pas générer, pour n'importe quel endroit de cette région, des surcotes correspondant à une période de retour 100 ans. L'estimation des événements extrêmes par la méthode du pooling permet d'examiner ce lien, à condition de tenir compte de la dépendance spatiale.

Tout récemment, WEISS et al. (2014b) ont proposé un modèle pour caractériser la dépendance spatiale, dans le cadre d'une analyse régionale effectuée par la méthode du pooling. En se basant sur la tendance qu'ont les sites à avoir un comportement similaire pendant une tempête, la dépendance est caractérisée à travers une fonction décrivant à la fois la propagation des tempêtes et leur intensité régionale. Ce modèle permet d'estimer la durée effective de l'échantillon régional, ou encore de comparer les périodes de retour locale et régionale d'une tempête.

L'objectif de cet article est d'appliquer ce modèle pour estimer des surcotes extrêmes de pleine mer, observées en 67 sites équipés de marégraphes sur les côtes françaises, britanniques et espagnoles de l’Océan Atlantique, de la Manche, de la Mer du Nord et de la Mer d'Irlande. 


\section{XIII ${ }^{\text {èmes }}$ Journées Nationales Génie Côtier - Génie Civil \\ Dunkerque, 2-4 juillet 2014}

\section{Méthodologie}

\subsection{Extraction des tempêtes}

Pour caractériser la dépendance spatiale éventuelle entre plusieurs sites, il est d'abord nécessaire de préciser ce qui définit la simultanéité des observations dans l'espace et le temps. Dans cet article, c'est l'échelle des événements physiques générant les extrêmes observés, les tempêtes, qui sert de référence pour définir la simultanéité des observations dans l'espace.

WEISS et al. (2014a) proposent une procédure pour détecter ces tempêtes, dans le cadre des événements maritimes extrêmes, en travaillant directement (et uniquement) avec la variable maritime d'intérêt: ici, la surcote de pleine mer. Les tempêtes sont alors définies comme les événements physiques générant des surcotes extrêmes de pleine mer en au moins un site de la zone d'étude. Afin de refléter leur propagation dans le temps et l'espace, un declustering spatio-temporel est utilisé : les extrêmes voisins dans le temps et l'espace sont supposés être générés par une même tempête.

Les extrêmes sont ici définis par les dépassements d’un seuil élevé de surcote de pleine mer. En chaque site, le seuil local est déterminé de façon à ce que celui-ci soit dépassé en moyenne $\lambda$ fois par an, i.e. $\lambda$ tempêtes y sont observées en moyenne chaque année.

\subsection{Analyse régionale par pooling}

Pour un site $i$ quelconque, soit $u_{i}$ le seuil dépassé en moyenne $\lambda$ fois par an et $X^{i}$ la variable des dépassements de ce seuil $u_{i}$. La Distribution de Pareto Généralisée (GPD) est la distribution naturelle pour modéliser des dépassements de seuil (DAVISON \& SMITH, 1990). Ainsi, $X^{i}$ est supposé suivre une loi GPD : $X^{i} \sim \operatorname{GPD}\left(u_{i}, \alpha_{i}, k_{i}\right)$, où $\alpha_{i}>0$ et $k_{i}$ sont, respectivement, un paramètre d'échelle et de forme, propres à chaque site. Le niveau de retour $T$ ans, i.e. la valeur dépassée en moyenne tous les $T$ années au site $i$, est

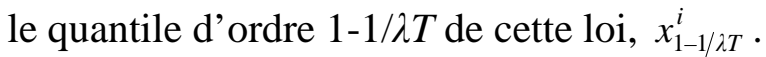

L'analyse régionale repose sur une hypothèse d'homogénéité : les observations de sites issus d'une région homogène sont supposées suivre la même loi de probabilité régionale, à un indice local près représentant les spécificités de chaque site. Pour une région homogène de $N$ sites, la variable normalisée $Y=X^{i} / u_{i}$ est ici supposée indépendante de $i$, de fonction de répartition (fdr) $F_{r}$. Cela implique que $Y$ suit la loi régionale $\operatorname{GPD}(1, \gamma, k)$, où le paramètre d'échelle régional vérifie $\gamma=\alpha_{i} / u_{i}$ et le paramètre de forme $k_{i}=k$ est constant dans la région. Le niveau de retour local $T$ ans au site $i$ s'écrit $x_{1-1 / \lambda T}^{i}=u_{i} y_{1-1 / \lambda T}$, où $y_{1-1 / \lambda T}$ est le niveau de retour régional $T$ ans (i.e. le quantile d'ordre $1-1 / \lambda T$ de $Y$ ).

La méthode du pooling, consistant à regrouper les observations normalisées des sites de la région dans un même échantillon, est utilisée pour estimer les paramètres $(\gamma, k)$ de la loi régionale. Cependant, les observations normalisées de la région ne doivent pas être directement toutes regroupées dans un même échantillon, à cause de la dépendance 


\section{Thème 1 - Hydrodynamique côtière}

spatiale qui peut affecter les estimations. Ce sont les tempêtes définies en section 2.1, qui, en contenant les empreintes spatiales des extrêmes issus d'un même événement, permettent de filtrer cette dépendance. Ainsi, pour se ramener à des données indépendantes, seul le maximum régional normalisé de chaque tempête est conservé. BERNARDARA et al. (2011) ont utilisé un filtre similaire, en ne retenant que l'observation maximale parmi les extrêmes apparus en moins de 72 heures dans la région. Si $n_{r}$ tempêtes sont observées dans la région sur la durée totale d’observation, l'échantillon régional est donc constitué des $n_{r}$ maxima régionaux normalisés. Les paramètres $(\gamma, k)$ sont ainsi estimés par maximum de vraisemblance pénalisé, à partir de l'échantillon régional.

\subsection{Durée effective régionale}

La méthode du pooling permet de définir une durée effective d'observation de $D_{\text {eff }}$ années. En particulier, elle traduit que $\lambda$ tempêtes sont observées en moyenne par an sur une période de $D_{\text {eff }}$ années. Cette quantité permet notamment d'évaluer les périodes de retour empiriques des tempêtes observées et de quantifier le gain d'une analyse régionale par rapport à une analyse locale.

$D_{\text {eff }}$ est étroitement liée à la dépendance spatiale, et est d'autant plus faible que cette dépendance est forte. Si les sites sont complètement indépendants, alors chaque tempête n’impacte qu'un seul site dans la région, quelle que soit son intensité. Dans ce cas, chaque observation apporte une nouvelle information, et $D_{\text {eff }}$ peut s'écrire comme la somme des durées locales d'observation : $D_{\text {eff }}=\sum d_{i}$, où $d_{i}$ est la durée d'observation au site $i$. A l'inverse, si les sites sont parfaitement dépendants, alors chaque tempête impacte tous les sites de la région. La durée locale d'observation d'un seul site

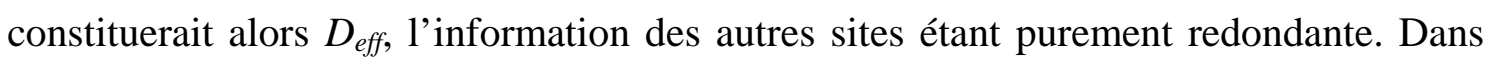
ce cas, $D_{\text {eff }}$ pourrait être par exemple résumée par la durée locale moyenne :

$D_{\text {eff }}=\sum d_{i} / N$.

Cependant, $D_{\text {eff }}$ se situe plus vraisemblablement entre ces deux situations extrêmes. WEISS et al. (2014b) proposent d'écrire, de façon plus générale, $D_{\text {eff }}=\varphi \sum d_{i} / N$, où $\varphi$ résume la dépendance régionale. $\varphi$, dont l'expression analytique relie la propagation des tempêtes et leur intensité, exprime la tendance des sites à avoir un comportement similaire durant une tempête impactant la région. $\varphi$ prend des valeurs comprises entre 1 (dépendance parfaite) et $N$ (indépendance totale), $N$ étant le nombre de sites dans la région. La dépendance régionale est d'autant plus forte que $\varphi$ est faible, indiquant dans ce cas que la plupart des sites sont impactés par une tempête, et ont tendance à réagir de façon similaire en termes d'extrêmes normalisés générés. Afin de comparer $\varphi$ entre plusieurs régions, l'effet de la taille de la région $N$ peut être enlevé en introduisant $\Phi=(N-\varphi) /(N-1)$, compris entre 0 (indépendance totale) et 1 (dépendance parfaite). 


\section{XIII ${ }^{\text {èmes }}$ Journées Nationales Génie Côtier - Génie Civil \\ Dunkerque, 2-4 juillet 2014}

WEISS et al. (2014b) montrent que $\varphi=\lambda_{r} / \lambda$, où $\lambda_{r}$ est le nombre annuel moyen de tempêtes dans la région et $\lambda$ le nombre annuel moyen de tempêtes en n'importe quel site. Ainsi, une estimation de $D_{\text {eff }}$ est donnée par :

$D_{\text {eff }}=n_{r} / \lambda$

où $n_{r}$ est le nombre de tempêtes observées dans la région.

\subsection{Lien entre la période de retour régionale et locale d'une tempête}

Soit $s$ une tempête quelconque et $x$ son intensité maximale (normalisée) observée dans la région. La période de retour régionale de la tempête $s, T_{r}$, est définie comme le temps moyen entre deux tempêtes impactant au moins un site de la région avec une intensité normalisée supérieure à $x$. La période de retour locale de $s, T$, est définie comme le temps moyen entre deux tempêtes impactant un site quelconque de la région avec une intensité normalisée supérieure à $x$. D’après WEISS et al. (2014b), $T_{r}$ et $T$ sont liées par la relation suivante :

$T_{r}=T / \varphi$

\section{Application à l'estimation des surcotes extrêmes de pleine mer}

\subsection{Données utilisées}

Les données brutes consistent en des séries temporelles de niveaux marins, observées en 67 sites équipés de marégraphes sur les côtes françaises, britanniques et espagnoles de l'Océan Atlantique, de la Manche, de la Mer du Nord et de la Mer d'Irlande. (figure 1). Les données françaises, fournies par le SHOM (Service Hydrographique et Océanographique de la Marine, France), sont disponibles sur le site internet de REFMAR (Réseaux de référence des observations marégraphiques). Les données espagnoles et britanniques sont respectivement fournies par les organismes IEO (Instituto Español de Oceanografía) et BODC (British Oceanographic Data Centre). Les niveaux marins des ports français et espagnols ont été observés au pas de temps horaire. Les données des ports britanniques ont quant à elles été observées au pas de temps horaire jusqu'en 1992, et au pas de temps 15 mn depuis 1993. Les séries temporelles, s'échelonnant de 1846 (Brest, France) jusqu'à 2011, ont une durée d'observation moyenne de 31 ans.

Les signaux de marée des 22 ports français (resp. 2 ports espagnols, La Corogne et Santander) ont été calculés par le logiciel SHOMAR, fourni par le SHOM. Les fichiers de données des 43 ports britanniques présentent la particularité de contenir à la fois les niveaux marins observés et les surcotes instantanées au même instant.

Pour calculer les séries de surcotes associées aux niveaux marins observés, comme les prédictions de marée sont données pour le temps présent, ces niveaux sont corrigés d'un éventuel eustatisme (altération à long terme des niveaux marins moyens, due à des effets géologiques ou climatiques). En chaque site, s'il existe une tendance significative 


\section{Thème 1 - Hydrodynamique côtière}

(détectée par régression linéaire) dans l'évolution temporelle des niveaux moyens annuels, elle est soustraite pour que ces derniers deviennent stationnaires.

La zone considérée étant fortement influencée par la marée, le risque de submersion côtière est plus important autour du moment de la pleine mer. Ainsi, seules les surcotes de pleine mer sont considérées (BERNARDARA et al., 2011 ; SIMON, 2007).

\subsection{Analyse régionale des surcotes extrêmes de pleine mer}

A partir des séries de surcotes de pleine mer aux 67 sites, 1706 tempêtes, correspondant au choix $\lambda=1$ ( 1 tempête en moyenne par an en chaque site), sont extraites par la procédure décrite en section 2.1. Par exemple, l'empreinte de la tempête Xynthia (27-28 février 2010) est représentée en figure 1a. Le marégraphe des Sables d'Olonne, qui s'est arrêté de fonctionner peu avant le passage de Xynthia, n’a pas mesuré la surcote de pleine mer associée à cette tempête.

Dans cet article, les régions homogènes sont déterminées comme étant les empreintes typiques des tempêtes (WEISS et al., 2014a), à partir de méthodes de classification sur un critère de propagation des tempêtes. Une tempête impactant une région donnée a donc tendance à $i$ ) impacter la plupart des sites de la région et ii) y rester à l'intérieur. 5 régions homogènes sont ainsi identifiées (figure $1 \mathrm{~b}$ ) : Golfe de Gascogne et Manche, Mer du Nord, Royaume-Uni Nord, Mer d'Irlande et Canal de Bristol. La méthode du pooling est ensuite appliquée pour chacune de ces régions séparément.

Le Tableau 1 contient les mesures de dépendance régionale des sections 2.3 et 2.4. Prenons l'exemple de la région 1 (Golfe de Gascogne et Manche), là où les tempêtes sont les plus fréquentes (environ 15 tempêtes en moyenne chaque année dans la région). La dépendance régionale y est la plus faible $(\Phi=0,49)$, en comparaison avec les autres régions : l'extension spatiale des tempêtes générant les surcotes extrêmes y est moindre. Pour chacun des 29 sites de cette région, $\lambda=1$ surcote extrême de pleine mer a été en moyenne observée chaque année, pendant une durée locale d'observation de 34 ans en moyenne. Le fait de regrouper ces extrêmes dans l'échantillon régional équivaut à une durée effective d'observation estimée à $D_{\text {eff }}=517$ ans. Ainsi, pour chaque site de cette région, l'extrapolation des extrêmes a désormais lieu à partir de 517 ans de données, et non 34 ans comme ce serait le cas (en moyenne) pour une analyse locale.

La figure 2 confronte les estimations empiriques et théoriques (loi GPD avec $\gamma=0,16$ et $k=0,12$ ) des niveaux de retour régionaux. Les trois plus fortes tempêtes observées dans cette région, respectivement la tempête Martin du 27 décembre 1999, celle du 16 octobre 1987 et la tempête Xynthia des 27-28 février 2010, sont notamment assez bien approchées par la loi GPD. Par ailleurs, $100_{r}=6,57$ ans signifie que tous les 6,57 ans en moyenne, une tempête génère en au moins un site de cette région une surcote de pleine mer de période de retour 100 ans (équation (2) pour $T=100$ ans). 


\section{XIII ${ }^{\text {èmes }}$ Journées Nationales Génie Côtier - Génie Civil \\ Dunkerque, 2-4 juillet 2014}

a)

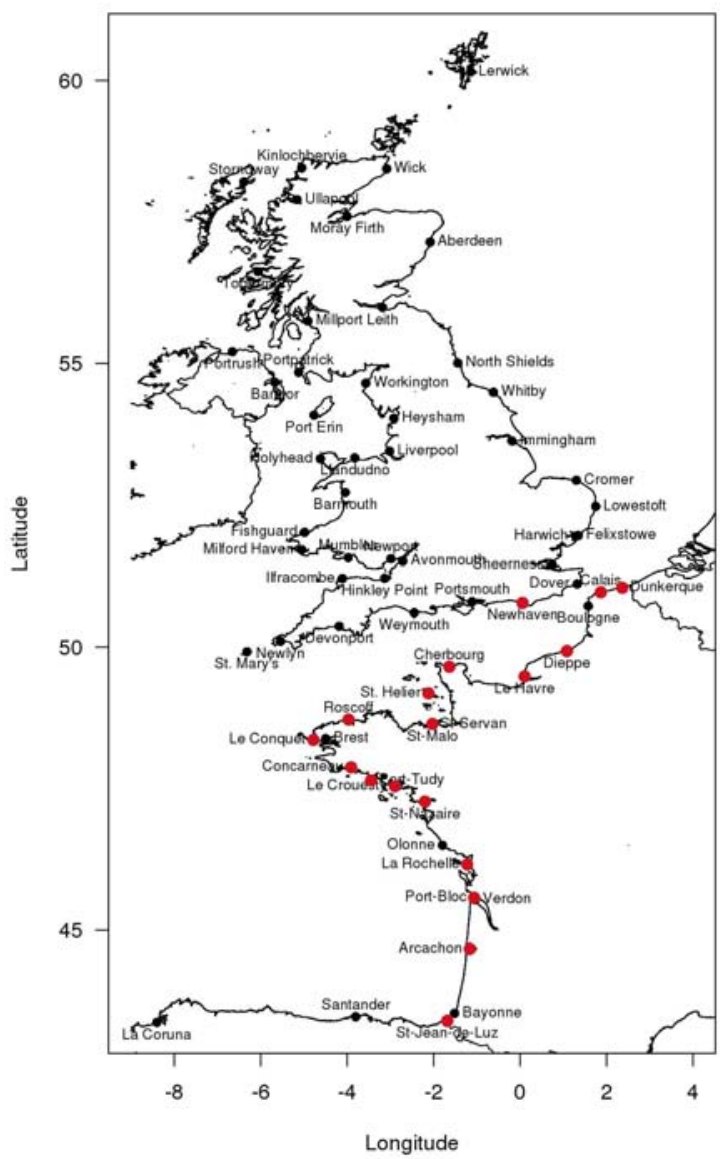

b)

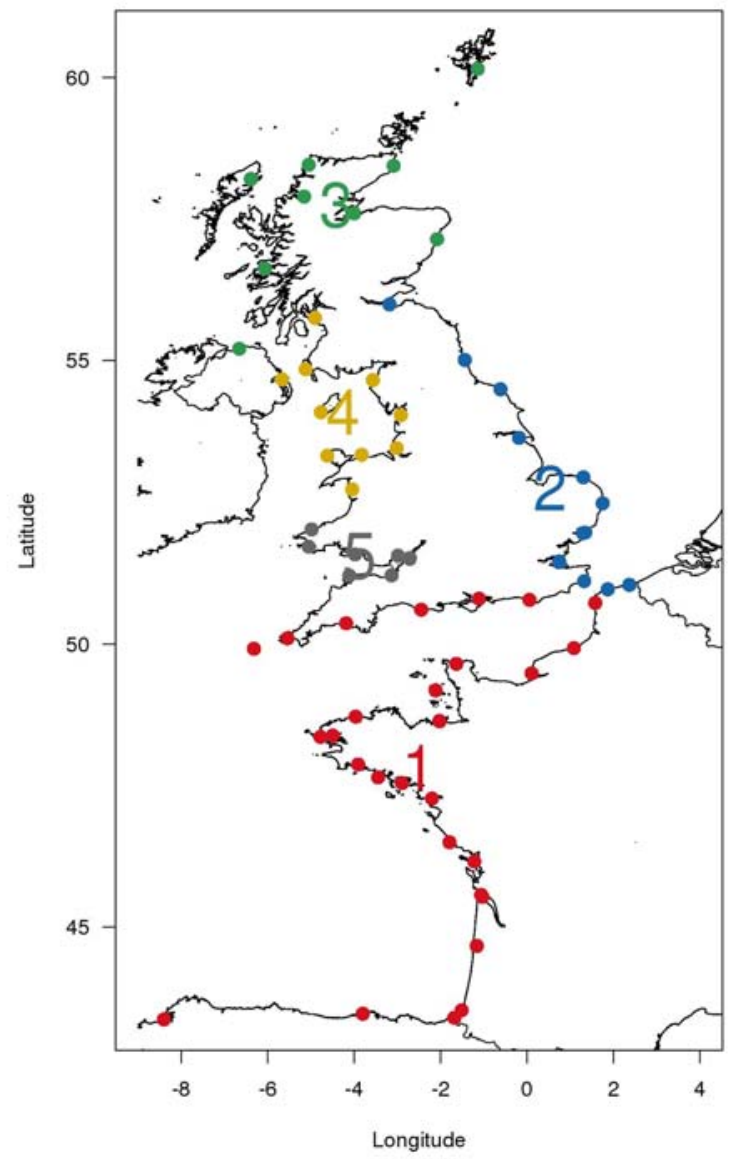

Figure 1. a) Empreinte de la tempête Xynthia du 27-28 février 2010 (sites impactés indiqués en rouge) b) Découpage proposé en 5 régions homogènes

Tableau 1. Mesures de dépendance régionale pour chaque région (nombre de sites entre parenthèses) : $\lambda_{r}$ est le nombre annuel moyen de tempêtes, $\Phi$ est le degré normalisé de dépendance régionale, $D_{\text {eff }}$ est la durée effective d'observation (en années, avec l'intervalle de confiance à 95\%) et $100_{r}$ est la période de retour régionale (en années) correspondant à une période de retour locale de 100 ans.

\begin{tabular}{llllll}
\hline Région & $\mathbf{1}(29)$ & $\mathbf{2 ( 1 2 )}$ & $\mathbf{3}(9)$ & $\mathbf{4}(10)$ & $\mathbf{5}(7)$ \\
\hline$\lambda_{\boldsymbol{r}}$ & 15,22 & 4,33 & 4,18 & 3,82 & 3,76 \\
$\boldsymbol{\Phi}$ & 0,49 & 0,70 & 0,60 & 0,69 & 0,54 \\
$\boldsymbol{D}_{\text {eff }}$ (ans) & $517[452,582]$ & $151[127,175]$ & $119[96,142]$ & $90[72,108]$ & $100[83,117]$ \\
$\mathbf{1 0 0}_{\boldsymbol{r}}$ (ans) & 6,57 & 23,08 & 23,94 & 26,17 & 26,60 \\
\hline
\end{tabular}




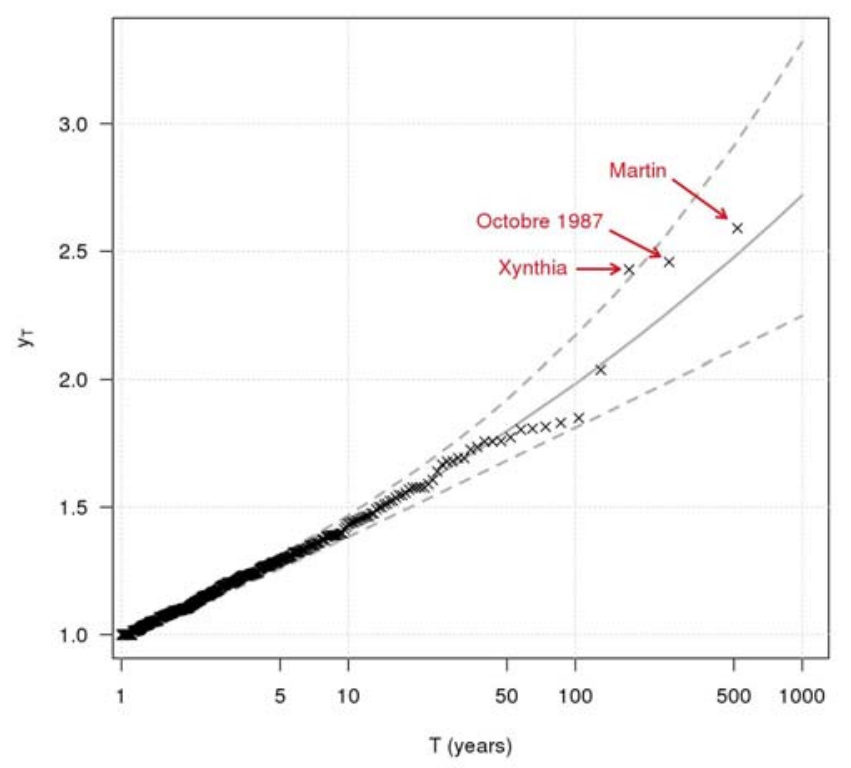

Figure 2. Evolution des niveaux de retour régionaux (normalisés) en fonction de la période de retour pour la région 1, avec intervalles de confiance bootstrap à 95\%.

Les croix correspondent aux observations de l'échantillon régional.

\subsection{Périodes de retour des tempêtes les plus intenses dans la région 1}

A titre d'exemple d'application de la méthode proposée, le Tableau 2 regroupe les estimations des périodes de retour des tempêtes les plus intenses observées dans la région 1 : la tempête Martin du 27 décembre 1999, celle du 16 octobre 1987 et Xynthia (27-28 février 2010). En particulier, l'estimation empirique (à partir des observations seulement) est différenciée de l'estimation théorique (issue de la modélisation par la loi GPD) de la période de retour. Ainsi, la surcote de pleine mer associée à la tempête Martin, qui a été la plus intense en termes de surcote normalisée observée, est estimée revenir dans la région tous les 34 ans (empiriquement) et 46 ans (théoriquement) en moyenne. Mais, à l'échelle d'un site quelconque de la région, cette surcote de pleine mer (en valeur normalisée) est estimée revenir tous les 518 ans (empiriquement) et 697 ans (théoriquement) en moyenne. En ce qui concerne la tempête Xynthia, la période de retour locale de la surcote de pleine mer normalisée correspondante est estimée à 173 ans (empiriquement) et 434 ans (théoriquement); sa période de retour régionale est quant à elle estimée à 11 ans (empiriquement) et 29 ans (théoriquement). On rappelle que ces estimations de périodes de retour ne considèrent que la variable "surcote de pleine mer". 


\section{XIII ${ }^{\text {èmes }}$ Journées Nationales Génie Côtier - Génie Civil \\ Dunkerque, 2-4 juillet 2014}

Tableau 2. Estimation des périodes de retour (en années) des tempêtes Martin (27 décembre 1999), du 16 octobre 1987, et Xynthia (27-28 février 2010), à l'échelle locale et régionale $\left(T_{\text {loc, }} T_{\text {reg }}\right)$, empirique et théorique $\left(T_{\text {emp }}, T_{\text {théo }}\right)$.

\begin{tabular}{llllll}
\hline Tempête & $\begin{array}{l}\text { Nombre de mesures } \\
\text { disponibles dans la } \\
\text { région }\end{array}$ & $\begin{array}{l}T_{\text {loc,emp }} \\
\text { (ans) }\end{array}$ & $\begin{array}{l}T_{\text {loc,théo }} \\
\text { (ans) }\end{array}$ & $\begin{array}{l}T_{\text {reg,emp }} \\
\text { (ans) }\end{array}$ & $\begin{array}{l}T_{\text {reg,théo }} \\
\text { (ans) }\end{array}$ \\
\hline Martin & $19 / 29(66 \%)$ & 518 & 697 & 34 & 46 \\
Octobre 1987 & $17 / 29(59 \%)$ & 259 & 474 & 17 & 31 \\
Xynthia & 23/29 (79\%) & 173 & 434 & 11 & 29 \\
\hline
\end{tabular}

\section{Discussion}

Le modèle développé, en utilisant l'information régionale, permet notamment de limiter le problème des courtes durées d'observation. Cependant, le problème des lacunes dans les données demeure (par exemple, lors d'opérations de maintenance/remplacement de marégraphes, ou à cause de pannes ou de défaillances pendant certaines tempêtes). Nous pensons cependant qu'une analyse régionale est plus robuste et tient mieux compte des éventuelles lacunes qu'une analyse locale. Par exemple, pendant la tempête Martin, une surcote importante s'est produite à La Rochelle, vraisemblablement de l'ordre de $2 \mathrm{~m}$, mais on ne dispose d'aucune mesure en ce site, à cause d'une panne de courant du marégraphe. Alors que la tempête Martin n’apparaît pas dans l'échantillon des surcotes mesurées à La Rochelle, la prise en compte de l'information régionale permet d'intégrer malgré tout cette tempête, via la mesure disponible au Verdon.

Par ailleurs, dans le modèle régional, l’indice local permet de représenter les spécificités de chaque site, par rapport aux caractéristiques régionales communes partagées par les sites de la région homogène. Ces spécificités locales peuvent être, par exemple, la configuration du trait de côte, la bathymétrie locale, ou encore des éventuels phénomènes de résonances, qui atténuent ou amplifient le signal régional de la surcote. Une amélioration possible du modèle régional serait de quantifier la part de ces différents effets de site intervenant dans l'indice local.

Par ailleurs, la zone d'étude a été impactée par une série de tempêtes assez remarquables au cours de l'hiver 2013-2014. Parmi elles, la tempête Xaver (5-8 décembre 2013) a notamment généré des surcotes de pleine mer de 1,12 $\mathrm{m}$ à Calais, 1,25 m à Dunkerque et $2 \mathrm{~m}$ à Lowestoft (DAUBORD (2014) et données BODC). En termes de valeurs normalisées, c'est à Calais que l'impact de la tempête Xaver a été le plus intense parmi ces 3 sites. Sur la base des résultats du modèle, la période de retour régionale (resp. locale) théorique de la surcote de pleine mer normalisée correspondant à la tempête Xaver est estimée à 11 ans (resp. 50 ans) dans la région 2 (Mer du Nord). 


\section{Conclusions}

Par rapport à une analyse statistique locale des extrêmes, l'analyse régionale permet de réduire les incertitudes sur les niveaux de retour estimés. Cependant, les tempêtes générant les surcotes extrêmes impactant généralement plusieurs sites, la dépendance spatiale se doit d'être prise en compte, en effectuant une analyse régionale par pooling. La méthodologie présentée dans cet article permet de quantifier et d'intégrer les effets de la dépendance entre les sites. Il devient possible d'estimer de façon plus réaliste la durée effective régionale d'observation, ou encore de lier les périodes de retour régionale et locale d'une tempête, permettant ainsi de mieux apprécier le risque à différentes échelles spatiales. Une application aux surcotes extrêmes de pleine mer est proposée, avec un zoom sur les tempêtes ayant généré les surcotes de pleine mer les plus importantes sur les façades Atlantique, Manche et Mer du Nord.

\section{Références bibliographiques}

BERNARDARA P., ANDREEWSKY M., BENOIT M. (2011). Application of the Regional Frequency Analysis to the estimation of extreme storm surges. Journal of Geophysical Research, 116, C02008, pp 1-11. http://dx.doi.org/10.1029/2010JC006229

DAUBORD C. (2014). Caractérisation de 7 évènements de tempête de l'automne-hiver 2013-2014 à partir des données disponibles au SHOM. Rapport d'étude SHOM $n^{\circ} 001 / 2014$.

DAVISON A.C., SMITH R.L. (1990). Models for exceedances over high thresholds. Journal of the Royal Statistical Society Series B (Methodological), Vol. 52(3), pp 393-442.

HOSKING J.R.M., WALLIS J.R. (1997). Regional Frequency Analysis. An approach based 687 on L-moments. Cambridge University Press.

SIMON B. (2007). La marée océanique côtière. Editions de l’Institut Océanographique.

WEISS J., BERNARDARA P., BENOIT M. (2014a). Formation of homogeneous regions for regional frequency analysis of extreme significant wave heights. Journal of Geophysical Research. http://dx.doi.org/10.1002/2013JC009668

WEISS J., BERNARDARA P., BENOIT M. (2014b). Modelling intersite dependence for regional frequency analysis of extreme marine events. Hal-00992636, http://hal.archivesouvertes.fr/hal-00992636. 